\title{
Analysis of ECRH switch on/off events in ASDEX Upgrade
}

\author{
V.F. Andreev ${ }^{1)}$, K.K. Kirov ${ }^{2)}$, A.V. Sushkov ${ }^{1)}$, Yu.N. Dnestrovskij ${ }^{1)}$, F. Leuterer ${ }^{2)}$, \\ G. Pereverzev $^{2)}$, K.A. Razumova ${ }^{1)}$, F. Ryter ${ }^{2)}$ and ASDEX Upgrade team ${ }^{2)}$ \\ 1) Nuclear Fusion Institute, RRC “Kurchatov Institute”, Moscow, Russia. \\ e-mail:roma@nfi.kiae.ru \\ 2) Max-Planck-Institute for Plasmaphysic, Garching, Germany. \\ e-mail: Krassimir.Kirov@ipp.mpg.de
}

\section{Introduction}

Heat and particle transport in tokamaks is an important issue in present and future fusion devices. A variety of transient processes occurring in fusion plasmas (limited time heating, operating mode transitions, ITB formation) requires investigation of the transport in nonstationary regimes.

In this paper we aim at understanding the transient features of the electron energy transport when off-axis, switched on/off ECRH power is applied. For that reason we use two different approaches to model the switch on/off events in ASDEX Upgrade, L-mode, low density $n_{e, 0}=2.5-3 \times 10^{19} \mathrm{~m}^{-3}$ no sawtoothing discharges, $B_{t}=-2.38 \mathrm{~T}, I_{p}=400 \mathrm{kA}$. In the first approach we use a local (or critical $\nabla T_{e} / T_{e}$ ) model of the transport coefficients and simulate the steady state and transient phases with the ASTRA code [1]. The second approach, the non-local model, uses directly the measured $T_{e}(\rho)$ changes to derive the evolution of the transport coefficients according to the solution of the inverse problem, COBRA code [2-4].

The evolution of the electron temperature profile $T_{e}(\rho)$ obeys the energy transport equation:

$\frac{3}{2} \frac{\partial}{\partial t}\left(n T_{e}\right)+\nabla\left(q_{e}+\frac{5}{2} T_{e} \Gamma_{e}\right)=p_{E C R H}+p_{\text {in }}-p_{\text {out }}+\Gamma_{e} \frac{\nabla\left(n T_{e}\right)}{n}$

where $n(\rho)$ is the plasma density and $\Gamma_{e}$ is the particle flux. The ECRH power deposition $p_{E C R H}(\rho)$ is approximated by a Gaussian with center $\rho_{0}$ and width $w_{0}$. Other input powers and the sinks are $p_{\text {in }}(\rho)$ and $p_{\text {out }}(\rho)$, respectively. In (1) the heat flux $q_{e}$ depends on the heat diffusivity $\chi_{e}$ and convective velocity $u_{e}$ :

$$
q_{e}=-n\left(\chi_{e} \nabla T_{e}-u_{e} T_{e}\right)
$$

\section{Critical $\nabla T_{e} / T_{e}$ model of the transport coefficients}

In this approach we suppose that the electron energy transport increases when the electron temperature gradient length $\nabla T_{e} / T_{e}=1 / L_{T e}$ exceeds a critical threshold $1 / L_{T e, c r}$. Based on this assumption we present $\chi_{e}$ empirically as a function of the local plasma parameters [5]:

$$
\chi_{e}=\chi_{e, 0}+q \lambda T_{e}^{3 / 2}\left(\nabla T_{e} / T_{e}-1 / L_{T e, c r}\right) H\left(\nabla T_{e} / T_{e}-1 / L_{T e, c r}\right)
$$

where $\chi_{e, 0}$ has a low value, close to neoclassical, and $\lambda$ is a parameter characterising $T_{e}(\rho)$ profile stiffness. The function $H\left(\nabla T_{e} / T_{e}-1 / L_{T e, c r}\right)$ is step-like nonlinear function, e.g. Heaviside function, which accounts for the enhanced transport when $\nabla T_{e} / T_{e}>1 / L_{T e, c r}$, and low transport for $\nabla T_{e} / T_{e}<1 / L_{T e, c r}$. From theoretical point of view two possible sources of turbulent transport feature such a dependence [6]: the Trapped Electron Modes (TEM) coupled with the Ion Temperature Gradient (ITG) modes and the Electron Temperature Gradient (ETG) modes. In our simulation we use $1 / L_{T e, c r}(\rho)$ profile, derived from the expression for the theoretical threshold for TEM driven turbulence $[7,8]$. The transport dynamics when $\nabla T_{e} / T_{e}$ exceeds the threshold can be investigated by applying switched on/off ECRH power and analysing $(i)$ the response of the electron temperature and ( $i i)$ the changes of the transport coefficients.

First we validate the approach based on the critical $\nabla T_{e} / T_{e}$ model by ASTRA simulations of 
Ohmic and ECRH heated steady-state discharges. By successful recovery of $T_{e}(\rho)$ profiles in these two regimes we obtain the parameter $\lambda$ and show the applicability of the model for simulation of switch on/off events.

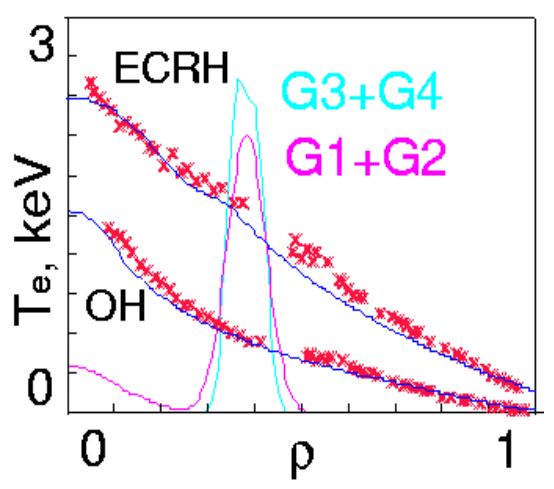

Figure 1. Electron temperature profiles from ASTRA simulations according to the critical $\nabla T_{e} / T_{e}$ model (blue lines) compared to the experimental ones (red points) in Ohmicaly heated $(\mathrm{OH})$ and in offaxis ECRH, gyrotrons 1-4 (G1-G4), heated discharges.

Good agreement, Fig.1, in the Ohmic phase is observed when we use a heat diffusivity according to (3) with $\lambda=1.2-1.5$. In the region $\rho<0.2$, one has $\nabla T_{e} / T_{e}<1 / L_{T e, c r}$ and the transport is close to neoclassical. However, to avoid very peaked $T_{e}(\rho)$ profile we have to suppose that $\chi_{e, 0}$ is $2-5$ times larger than neoclassical. In a broad range, $0.2<\rho<0.5, \nabla T_{e} / T_{e}$ is close to $1 / L_{T e, c r}$, which can be explained with the on-axis nature of the Ohmic heating. For $\rho>0.5$ the transport is turbulent since $\nabla T_{e} / T_{e}>1 / L_{T e, c r}$. In the ECRH phase good agreement, Fig.1, is observed with the same values for $\chi_{\mathrm{e}, 0}$ and $\lambda$ and if one assumes a few percents, $2-3 \%$, additional central heating, e.g. spurious ECRH or some heat pinch [9]. The center of ECRH power deposition was taken from TORBEAM [10] calculations with a deposition width $w_{0}=2-3 \mathrm{~cm}$. The $\nabla T_{e} / T_{e}$ model correctly reproduces the feature of off-axis ECRH to create low transport inside and enhanced transport outside the deposition, Fig.2, red dashed line.

$\# 15600, \mathrm{ECRH}$ switch off at $3.54 \mathrm{~s}$
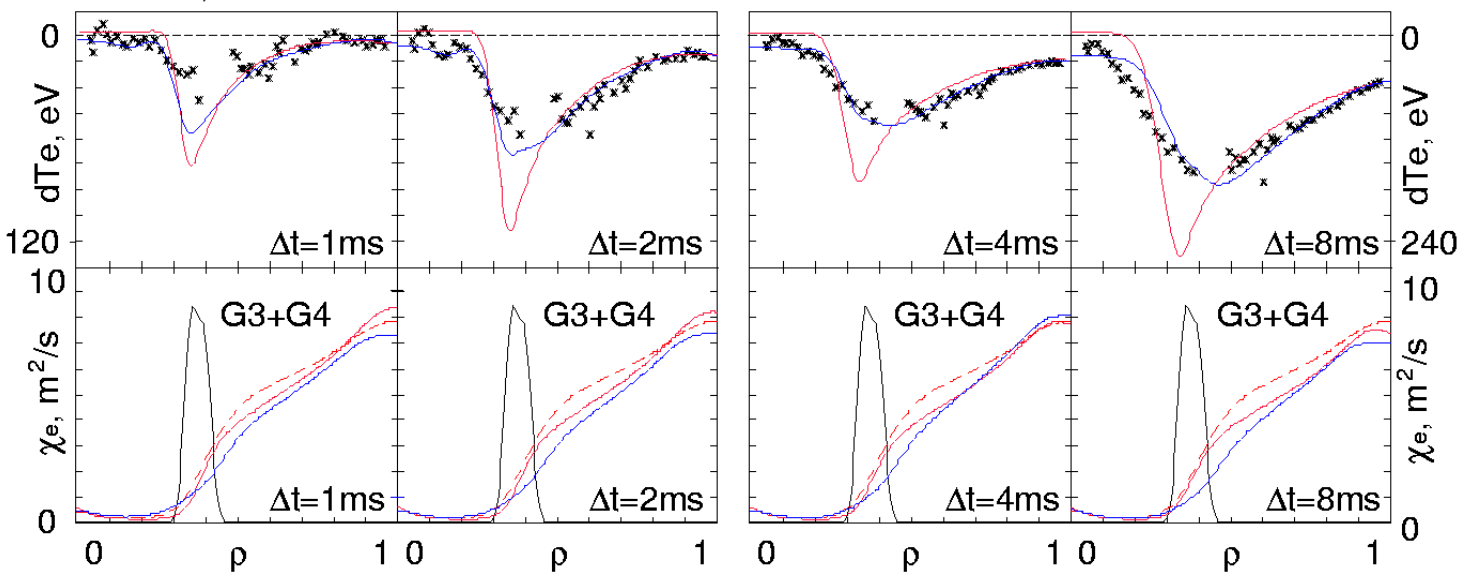

Figure 2. Changes of the temperature $d T_{e}$ and heat diffusivity $\chi_{e}$ for $1,2,4$ and $8 \mathrm{~ms}$ after switching off the ECRH power from gyrotrons 3 and 4 . The results from ASTRA calculations with critical $\nabla T_{e} / T_{e}$ model of the heat diffusivity are given by red lines and with $\chi_{e}$ evolution from non-local model are given in blue. The red dashed line is the steady-state $\chi_{e}$ profile in both models and points correspond to experimental results for $d T_{e}$.

The transient process of switching off ECRH power shows good match between the simulated temperature drop $d T_{e}$ and the measured one outside from the ECRH deposition, while in central region one needs somewhat different transport, Fig.2. The $d T_{e}$ changes are better reproduced in the switch on case, Fig.3. If we exclude the very instant moment, $\Delta t=1 \mathrm{~ms}$, after switching on the ECRH power the good match between the simulated jump of the electron temperature and the experimental one for $\rho>0.3$ confirms the applicability of $\nabla T_{e} / T_{e}$ model in the description of the transient phase of switching on the ECRH power.

The poor agreement for $\rho<0.3$, i.e. inside from the ECRH deposition, in both cases of switching on and switching off the ECRH power can be explained with impossibility of Eq.(3) to describe the changes of $\chi_{e, 0}$ term in the investigated transient processes. This can be seen in Fig. 2 where following the deviation of $\chi_{e}$ (red lines) from its steady-state value (red dashed line) one can see that the heat diffusivity does not change in the region $\rho<0.4$. 

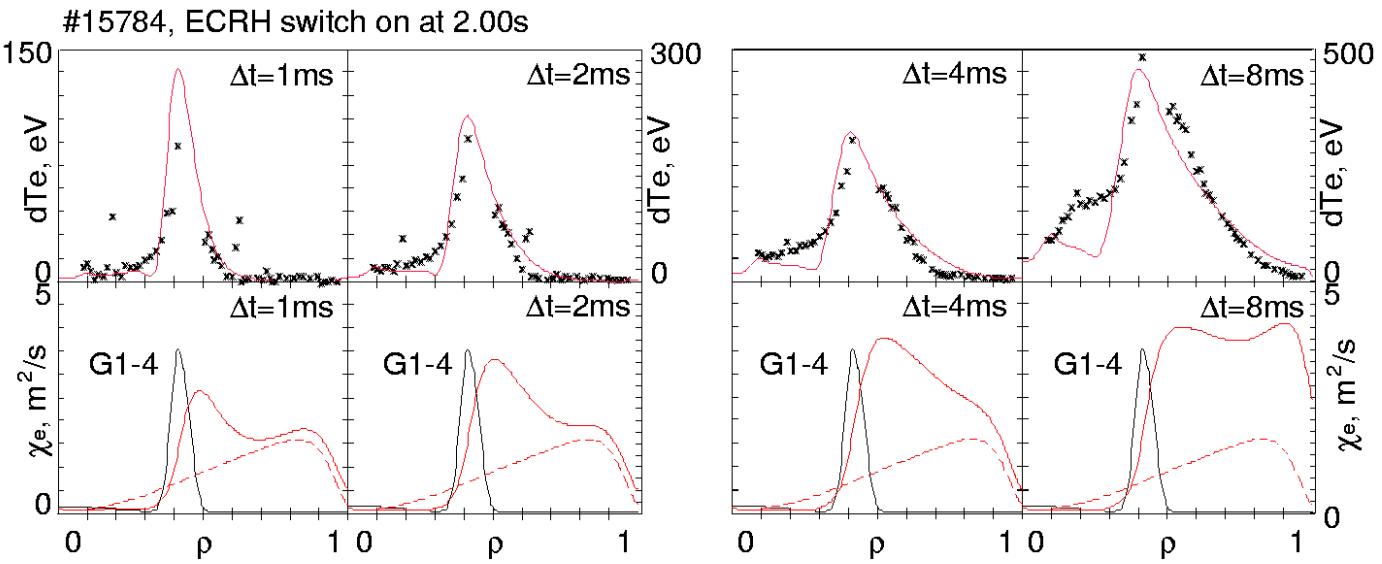

Figure 3. The increase of the electron temperature $d T_{e}$ for 1,2,4 and $8 \mathrm{~ms}$ after switching on the ECRH power. The results from ASTRA calculations with critical $\nabla T_{e} / T_{e}$ model of the heat diffusivity, equ. (3), are given by red lines, the points correspond to experimental results for $d T_{e}$.

\section{Non-local model of the transport coefficients}

We use the following equations for the description of transient process by non-local model. To write the steady state heat conductivity equation before the ECRH switching on/off and the heat conductivity equation for transition process after ECRH switching on/off. For the transient process one can represent the density $n(r, t)$ and temperature $T(r, t)$ as a sum of stationary values $n^{S}(r), T^{S}(r)$ and time-dependent variations $\widetilde{n}(r, t)$ and $\widetilde{T}(r, t)$ :

$$
n(r, t)=n^{S}(r)+\widetilde{n}(r, t), \quad T(r, t)=T^{S}(r)+\widetilde{T}(r, t) .
$$

Since the ECRH power is mainly input into the electrons, the electron temperature changes much faster than density. This means that the relative density variation is much less than the relative temperature variation. In this report the transient process is considered for the short time intervals (10-20 ms), then the density variation can not be taken into account $n(r, t)=n^{S}(r)$. We suppose that the heat diffusivity $\chi_{e}$ and the convective velocity $u_{e}$ are nonlocal functions of plasma parameters. This means that changes of $\chi_{e}$ and $u_{e}$ in any point of plasma radii may occur although the local values of the density and temperature in the given point are not changed. We present $\chi_{e}$ and $u_{e}$ at transient process in the form:

$$
\begin{aligned}
& \chi_{e}(r, t)=\chi_{e}^{S}(r)+\tilde{\chi}_{e}(r, t), \quad u_{e}(r, t)=u_{e}^{S}(r)+\tilde{u}_{e}(r, t), \\
& \tilde{\chi}_{e}(r, t)=\left\{\begin{array}{ll}
0, & t \leq t_{S} \\
\tilde{\chi}_{e}(r, t), & t>t_{S}
\end{array}, \quad \tilde{u}_{e}(r, t)=\left\{\begin{array}{ll}
0, & t \leq t_{S} \\
\tilde{u}_{e}(r, t), & t>t_{S}
\end{array} .\right.\right.
\end{aligned}
$$

Really we assume that the variations $\tilde{\chi}_{e}$ and $\tilde{u}_{e}$ can change faster than the characteristic time of transient process. But, relations $\tilde{\chi}_{e} / \chi_{e}^{S}$ and $\tilde{u}_{e} / u_{e}^{S}$ are not small in respect to unity. The whole set of these assumptions we call as non-local model of transport coefficients [2-3]. By subtraction the steady state heat conductivity equation from the heat conductivity equation for transition process, using (4-5), one can get the equation for the temperature variation $\widetilde{T}$ :

$$
\begin{aligned}
& \frac{3}{2} \frac{\partial}{\partial t}\left(n^{S} \widetilde{T}\right)=\frac{1}{r} \frac{\partial}{\partial r}\left[r n^{S}\left(\chi_{e}^{S}+\widetilde{\chi}_{e}\right) \frac{\partial \widetilde{T}}{\partial r}\right]-\frac{1}{r} \frac{\partial}{\partial r}\left[r n^{S} \widetilde{u}_{e} \widetilde{T}\right]+P_{E C}+ \\
& +\left[\left(P_{O H}-P_{O H}^{S}\right)+\left(Q-Q^{S}\right)\right]+\frac{1}{r} \frac{\partial}{\partial r}\left(r n^{S} \widetilde{\chi}_{e} \frac{\partial T^{S}}{\partial r}\right)-\frac{1}{r} \frac{\partial}{\partial r}\left(r n^{S} \widetilde{u}_{e} T^{S}\right), \\
& \partial \widetilde{T} / \partial r(r=0, t)=0, \widetilde{T}(r=1, t)=0, t>t_{S}, \widetilde{T}\left(r, t=t_{S}\right)=0,0<r<1 .
\end{aligned}
$$

The Eq. (6) contains terms which are determined by the steady state electron temperature $T^{S}$ and the variation of transport coefficients $\tilde{\chi}_{e}, \tilde{u}_{e}$, but they do not depend on the temperature 
variation $\widetilde{T}$. Note, that equ. (6) is not linearized equation. In equ. (6) following parameters are unknown: the stationary values of electron heat diffusivity $\chi_{e}^{S}$ and convective velocity $u_{e}^{S}$, their variations $\tilde{\chi}_{e}$ and $\widetilde{u}_{e}$ during transient phase and the ECRH power profile $P_{E C}(r)$.

The inverse problem is formulated for transient process after switching on/off ECRH in ASDEX Upgrade. At that we assume that after ECRH switch on/off either the heat diffusivity or the convective velocity are changed only. Assuming the electron temperature is known at a few radial points and at several time moments, the evolution of $\chi_{e}$ profile or $u_{e}$ profile and the heat source are found from the following condition: the discrepancy functional (a weighted sum of squares of the differences between the calculated temperature and the experimental values) has a minimum value. The method of the inverse problem solution includes the parametric presentation of the transport coefficients and the ECRH power profile. An example is shown in Fig.4, where the transient process was analysed by COBRA either assuming change in $\chi_{e}$ or in $u_{e}$. Calculations show that the heat diffusivity or the convective velocity (heat pinch) change from the steady state value very fast after ECRH switch on/off.

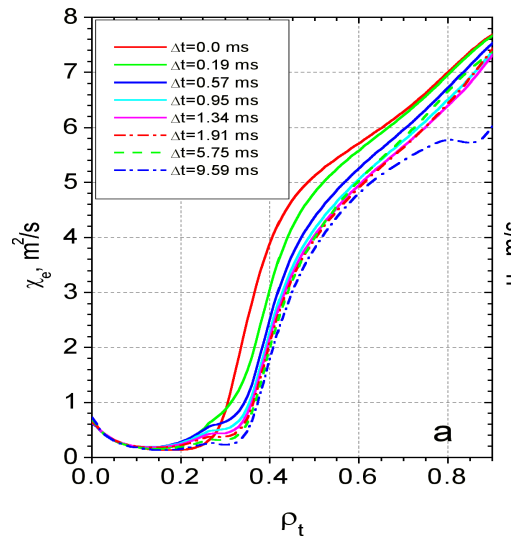
ASDEX Upgrade, \#15600, off-axis ECRH switch off, $t=3.54 \mathrm{~s}$

Figure 4. The heat diffusivity (a), or the convective velocity (b) for different time moments from COBRA calculations for transient process after off-axis ECRH switching off and ECRH power profile (c).

\section{Summary and conclusions}

The ASTRA simulations with heat diffusivity from the non-local and the critical $\nabla T_{e} / T_{e}$ model are given in Fig. 2 by blue and red lines, respectively. In the case of switching off the ECRH power the temperature drop $d T_{e}$ obtained from the non-local model matches better the experimental data for $\rho<0.5$. This may be due to the fact that in the COBRA analysis the transport in this region is treated as time dependent, while in the ASTRA simulations with the critical $\nabla T_{e} / T_{e}$ model this is the region of the transition from low to turbulent transport and $\chi_{e}$ remains constant in time there. Following the changes of $\chi_{e}$ (blue lines in Fig.2) after switching off ECRH power one sees that significant changes of the heat diffusivity from its steady-state value (dashed line) occur in the region $0.3<\rho<0.5$. This explains better recovery of the experimental data in this case. Outside of ECRH deposition $(\rho>0.5)$ both models give similar good results and predict the changes of $\chi_{e}$ in agreement.

\section{References:}

1. Pereverzev G et al 1991 Rep. IPP 5/42, MPI for Plasmaphysic, Garching

2. Andreev V F et al 2002 Plasma Physics Reports 28, No.5, 367.

3. Andreev V F et al $20029^{\text {th }}$ EU-US TTF Workshop, Cordoba, Spain, http://www-fusion.ciemat.es/ttf2002/

4. Andreev V F, Dnestrovskij Yu N, Popov A M 1993 Nucl. Fusion 33499.

5. Ryter F et al $200219^{\text {th }}$ IAEA Fusion Energy Conference, Lyon, France.

6. Weiland J 2000 Collective Modes in Inhomogeneous Plasma, IOP Publishing, London.

7. Tardini G et al 2002 Nucl. Fusion 42 L11.

8. Weiland J et al 1989 Nucl. Fusion 291810.

9. Mantica P et al $200330^{\text {th }}$ EPS, St Petersburg, Russia.

10. Poli E et al 2001 Comput. Phys. Commun. 13690. 